\title{
The energy absorbing properties of bamboo-based structures
}

\begin{abstract}
A series of quasi-static and dynamic tests on bamboo-based honeycomb and bambooreinforced foam structures were carried out to investigate their energy absorbing characteristics and the related failure modes. Here, the tube damage shows the typical buckling (bulging) and top surface splitting failure. The results on the individual tubes show that the energy absorbing capacity increases as the diameter-to-thickness ratio decreases. Simple analytical models were also developed to predict the peak load and the corresponding displacement, with reasonably good correlation to the experimental results, in spite of the inherent variability of the bamboo tubes. It was found that different dynamic responses of the individual bamboo tubes and grouped bamboo tubes in the honeycomb cores affect their energy absorbing capabilities. The composite structure made from bamboo and foam shows that the embedded tube exhibits a higher energy absorption capacity than the sum of the individual foam and bamboo, due to the constraint offered by the foam.
\end{abstract}

Keyword: Bamboo-based honeycomb; Bamboo tube; Mechanical properties; Analytical model; Energy absorption 